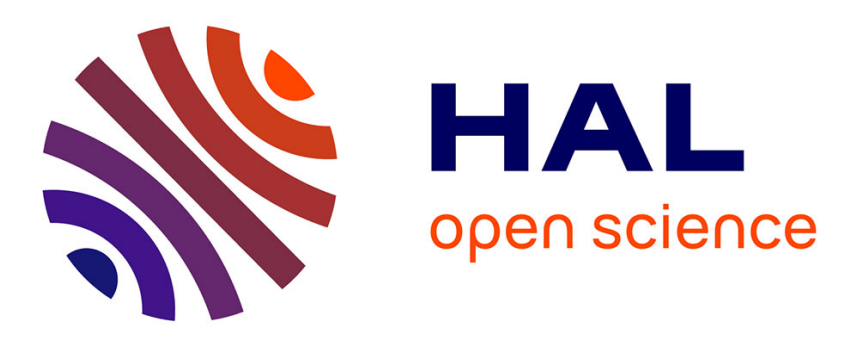

\title{
Photothermal frequency-domain depth profilometry of a discrete inhomogeneous surface layer on homogeneous substrate
}

\author{
F. Funak, A. Mandelis, M. Munidasa
}

\section{- To cite this version:}

F. Funak, A. Mandelis, M. Munidasa. Photothermal frequency-domain depth profilometry of a discrete inhomogeneous surface layer on homogeneous substrate. Journal de Physique IV Proceedings, 1994, 04 (C7), pp.C7-95-C7-98. 10.1051/jp4:1994724 . jpa-00253252

HAL Id: jpa-00253252

https://hal.science/jpa-00253252

Submitted on 1 Jan 1994

HAL is a multi-disciplinary open access archive for the deposit and dissemination of scientific research documents, whether they are published or not. The documents may come from teaching and research institutions in France or abroad, or from public or private research centers.
L'archive ouverte pluridisciplinaire $\mathbf{H A L}$, est destinée au dépôt et à la diffusion de documents scientifiques de niveau recherche, publiés ou non, émanant des établissements d'enseignement et de recherche français ou étrangers, des laboratoires publics ou privés. 


\title{
Photothermal frequency-domain depth profilometry of a discrete inhomogeneous surface layer on homogeneous substrate
}

F. Funak, A. Mandelis and M. Munidasa

Photothermal and Optoelectronic Diagnostics Laboratory, Department of Mechanical Engineering and Manufacturing Research Corporation of Ontario, University of Toronto, Toronto, Ont. Canada, M5S IA4

\begin{abstract}
A technique for obtaining thermal diffusivity depth profiles of inhomogeneities in twolayered systems is discussed. The method uses incremental differences in the photothermal signal frequency response of a sample with the inhomogeneous surface layer compared to a homogeneous reference sample. Applications to machined steel samples are reported.
\end{abstract}

\section{INTRODUCTION}

In recently published work, a continuum approach to the photothermal depth profilometric problem of inhomogeneous, semi-infinite condensed phases was found to be successful in reconstructing depth-dependent thermal diffusivity profiles, using photoacoustic gas-cell [1] or infrared photothermal radiometric (PTR) [2] detection. The main feature of those approches was the measurement of the thermal-wave frequency response (amplitude and phase) of a continuously inhomogeneous solid sample, normalized by the response of a semi-infinite homogeneous reference sample.

In this work, the earlier formulation is extended to the technologically important case of a twolayered solid consisting of a semi-infinite, homogeneous substrate supporting an inhomogeneous surface layer of thickness L. Such configurations arise, for example, in plasma spraying/coating of metals.

\section{THEORETICAL}

Figure 1 shows the assumed (ideal) geometry. The a.c. temperature profile in each region: air (g); investigated layer (IL or s); and substrate (b) is generated by a modulated laser beam with sufficient lateral extent so as to create a one-dimensional thermal-wave profile in the structure of Fig.1. The intensity of the beam is modulated at an angular frequency $\omega$. The a.c. temperature fields are:
(a) Gas:$$
T_{g}(x, \omega)=\Theta \cdot e^{\sigma x}
$$$$
; x \leq 0
$$
(b) Solid:$$
T_{s}(x, \omega)=T_{s}(0) \cdot \sqrt{R(x)} \cdot\left[C_{1} e^{H(x)}-C_{2} e^{-H(x)}\right]
$$$$
; 0 \leq x \leq L
$$$$
\text { (c) Bulk substrate; } T_{b}(x, \omega)=U \cdot e^{-\sigma(x-L)}
$$ 


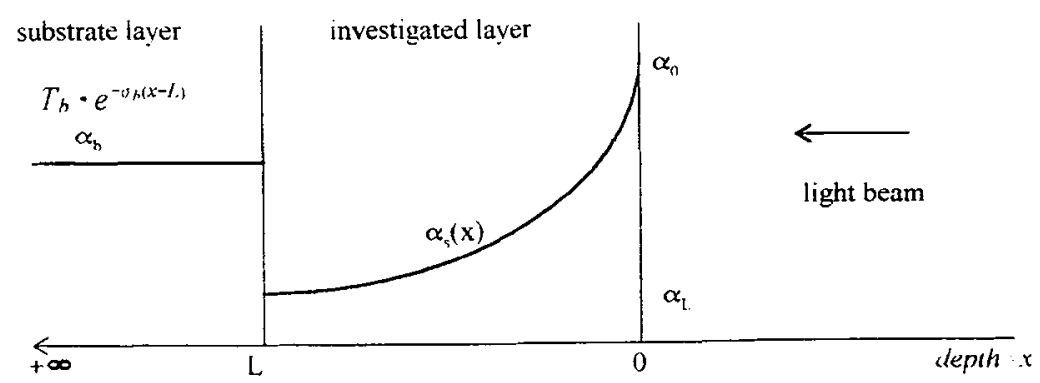

Figure 1. One-dimensional geometry of a discrete inhomogeneous surface layer on a homogeneous semi-infinite substrate.

Eqs.(1a), (1c) are standard solutions to the thermal-wave equation in homogeneous semi-infinite media [3]. Eq.(1b) is the result of a treatment of the inhomogeneous layer thermal-wave field in terms of the Hamilton-Jacobi formulation of the problem [4]. In Eqs. (1) the following definitions were made:

$$
\begin{array}{ll}
\omega=2 \pi f & : \text { angular modulation frequency of the optical intensity } \\
\sigma_{j}(\omega) \equiv(1+1) \sqrt{\frac{\omega}{2 \alpha_{j}}} ; \alpha_{j} & : \text { diffusivity of the } \mathrm{j} \text {-th (homogeneous) medium (j:g,b) } \\
R(x) \equiv \frac{e_{s}(0)}{e_{s}(x)} \quad ; e_{s}(y) & : \text { thermal effusivity of the IL at } x=y \\
L & : \text { thickness of the inhomogeneous layer } \\
H(x) \equiv \int_{0}^{x} \sigma_{s}(y, \omega) d y & : \sigma_{s} \text { is defined analogously to } \sigma_{j}: \sigma(y, \omega)=(1+I) \sqrt{\frac{\omega}{2 \alpha_{s}(y)}}
\end{array}
$$

where $\alpha_{s}(\mathrm{y})$ is the thermal diffusivity of the IL at $\mathrm{x}=\mathrm{y}, C_{b} C_{3} \Theta, T_{s}(0)$ and $U$ are constants of integration to be determined by boundary and limiting conditions. The IL is assumed to be strictly opaque, i.e. its optical penetration depth is much shorter than the shortest thermal diffusion length, $\mu_{s}(\omega)=\left(2 \alpha_{s} / \omega\right)^{1 / 2}$, in this layer. At the interface $\mathrm{x}=\mathrm{L}$, continuity of temperature and heat flux (standard treatment) is assumed. To proceed further, an expression for the (assumed arbitrary) $\alpha_{\mathrm{s}}(\mathrm{x})$ profile must be used, in order to obtain explicit expressions for $H(x)$. A simulated function which is convenient in simplifying the integration involved is $[4,5]$

$$
\alpha_{s}(x)=\alpha_{0}\left(\frac{1+\Delta e^{-q x}}{1+\Delta}\right)^{2}
$$

such that $\alpha_{s}(L)=\alpha_{L}, \alpha_{s}(0)=\alpha_{0}$ and

$$
\Delta=\frac{1-\sqrt{\alpha_{L} / \alpha_{0}}}{\sqrt{\alpha_{L} / \alpha_{0}}-e^{-q L}}
$$

The parameter $q$ is a constant to be determined from experimental data of photothermal signals (amplitude and phase) vs. frequency $\omega$. Insertion of Eqs. (2) and (3) in the integral for $H(L)$ yields: 


$$
H(L)=(1+i) \sqrt{\frac{\omega}{2 \alpha_{L}}}\left[\frac{1-e^{-q L}}{1-\sqrt{\left(\alpha_{0} / \alpha_{L}\right)} e^{-q L}}\right]\left[L-\frac{1}{2 q} \ln \left(\frac{\alpha_{0}}{\alpha_{L}}\right)\right]
$$

Finally, writing out the resulting thermal-wave field for a non-homogeneous IL gives:

$$
T_{s}(0)=\frac{Q_{0}}{k_{s}(0) \sigma_{s}(0)}\left[\frac{1+\gamma_{b s} e^{-2 H(L)}}{1-\gamma_{b s} e^{-2 H L)}}\left(1+G_{L}(\omega)\left[\frac{1+\gamma_{b s} e^{-2 H(L)}}{1-\gamma_{b s} e^{-2 H(L)}}\right]\right)\right]
$$

where

$$
G_{L}(\omega)=\frac{1}{4} \sqrt{R(L)} \exp \left[-\frac{(1+i) \sqrt{\omega}}{2 \sqrt{2} q} \cdot \frac{1}{\sqrt{\alpha_{L}}} \ln \left(\frac{\alpha_{0}}{\alpha_{L}}\right)\right]
$$

The coupling coefficient $\gamma_{b s}$ at the back interface is given by

$$
\gamma=\frac{1-b_{b s}}{1+b_{b s}} \text { where } b_{b s}=\frac{\sigma_{b} k_{b}}{\sigma_{s}(L) k_{s}(L)}=\frac{k_{b} \sqrt{\alpha_{s}(L)}}{k_{s}(L) \sqrt{\alpha_{b}}} .
$$

\section{COMPUTATIONAL AND NUMERICAL CONSIDERATIONS OF THE INVERSE PROBLEM ALGORITHM}

The surface temperature response to the incident light beam on the investigated system (sample) is normalized by the surface temperature response to the same beam of the same frequency on a semi-infinite homogeneous material (reference). This gives for each frequency a data-pair: amplitude ratio and phase difference between sample and reference. The normalizing procedure is necessary for the correct accounting of all frequency dependencies in the experimental system, other than that due to the IL (ie. the instrumental transfer function). Theoretical values of the datapair are calculated by Eq.(5):

$$
|M(\omega)| \cdot e^{i \Delta \phi(\omega)}=\frac{k_{r e f} \sigma_{r e f}}{k_{s}(0) \sigma_{s}(0)} \cdot\left[\frac{1+\gamma_{b s}(L) e^{-2 H L)}}{1-\gamma_{b s}(L) e^{-2 H(L)}}\left(1+G(\omega)\left[\frac{1+\gamma_{b s}(L) e^{-2 H(L)}}{1-\gamma_{b s}(L) e^{-2 H L}}\right]\right)\right]
$$

$|\mathrm{M}(\omega)|$ is the amplitude ratio and $\Delta \phi(\omega)$ is the phase difference at angular frequency $\omega$. To be able to calculate thermal diffusivity $\alpha_{s}(x)_{(j)}$ and depth $x_{(j)}$ corresponding to frequency $\omega_{(j)}$, one has to find the unknown parameters $\alpha_{0(j)}$ and $\mathrm{q}_{(j)}$. For this task the two-dimensional Broyden's method [4] was used. The procedures had to be customized for Eq.(7) to avoid run time errors and singularities in Broyden's two-dimensional line search procedure. The method looks for $\alpha_{0(j)}$ and $\mathrm{q}_{\mathrm{j}}$ to satisfy the minimum condition $\left|\mathrm{M}_{\text {exp }}\left(\omega_{\mathrm{j}}\right)\right|-\left|\mathrm{M}_{\text {theor }}\left(\omega_{\mathrm{j}}\right)\right|=0$ and $\Delta \phi_{\text {exp }}\left(\omega_{\mathrm{j}}\right)-\Delta \phi_{\text {theor }}\left(\omega_{\mathrm{j}}\right)=0$ for each angular frequency $\omega_{j}$. Search start from the lowest frequency $\omega_{1}$.

In the following examples we tested the fidelity of the foregoing method for the reconstruction of known thermal diffusivity depth-profiles. Figure 2 shows four simulated profiles and the corresponding reconstructions in a geometry consisting of a thin solid layer on an arbitrary homogeneous semi-infinite substrate. The reconstructions are in excellent agreement with the original profiles. 


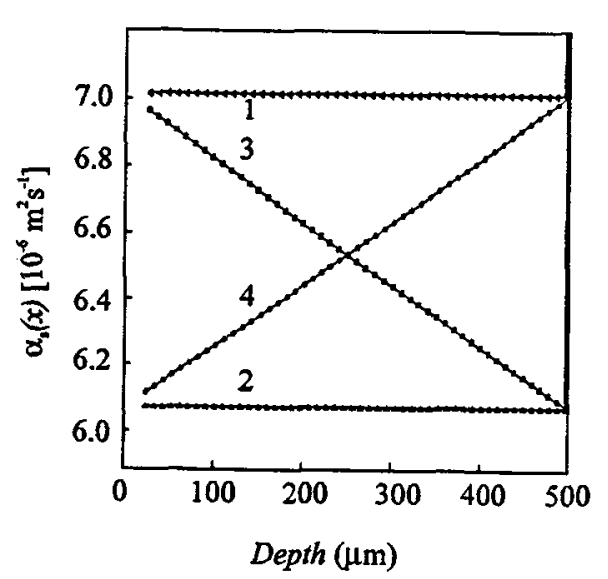

Figure 2. Simulated (solid lines) and reconstructed (triangles and squares) profiles of two homogeneous $(1,2)$ and two inhomogeneous $(3,4)$ samples.

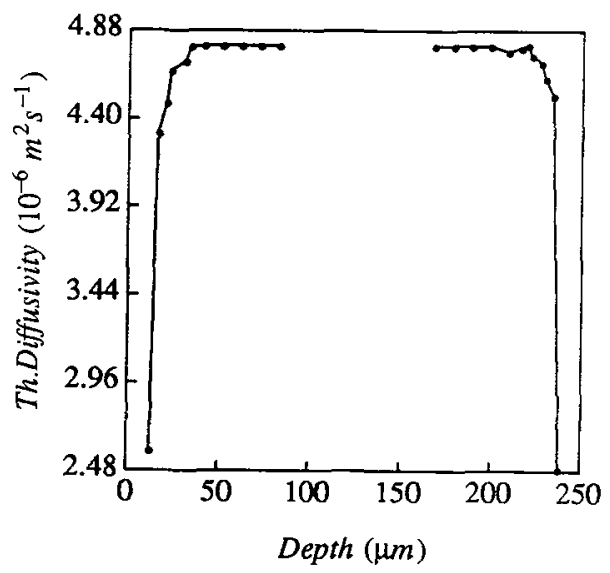

Figure 3. Reconstructed profile from a machined steel plate of thickness $\mathrm{L}=250 \mu \mathrm{m}$.

\section{EXPERIMENTAL}

The semi-infinite approximation to the foregoing algorithm was then used to obtain the thermal diffusivity profiles of machined low carbon stainless steel plates of different thicknesses using PTR detection [2]. Air was used as bulk sustrate which allowed probing, thermal diffusivity reconstruction and depth-profile generation from both sides of the sample. Figure 3 shows the reconstructed profile for the $250 \mu \mathrm{m}$ thick sample. The machining process appears to disturb the thermophysical properties of low-carbon steel down to depth of $c a .30 \mu \mathrm{m}$, while the material bulk remains undisturbed at approx. $4.8 \times 10^{-6} \mathrm{~m}^{2} \mathrm{~s}^{-1}$ in good agreement with literature values.

In conclusion, the present method can successfully reconstruct diffusivity profiles of discrete inhomogeneous layers on homogeneous substrates. In particular stainless steel thermal diffusivity profiles due to machinig process were obtained clearly showing the near-surface depth range of machining damage for the first time. Further investigations are now possible on the effect of machining on material properties (thermophysical and mechanical), possibly leading to a better maufacturing control of the process.

\section{REFERENCES}

[1]. T-C. Ma, M. Munidasa and A. Mandelis, J.Appl.Phys. 71, 6029-6035 (1992).

[2]. M. Munidasa, T-C. Ma, A. Mandelis, S.K. Brown and L. Mannik, Mat.Sci.Eng. A 159, 111-118 (1992).

[3]. H.S. Carslaw and J.C. Jaeger, "Conduction of Heat in Solids," 2nd ed., (Clarendon, Oxford, (1960).

[4]. A. Mandelis, S.B. Peralta and J. Thoen, J.Appl.Phys. 70, 1761-1770 (1991).

[5]. A. Mandelis, E. Schoubs, S.B. Peralta and J. Thoen, J.Appl.Phys. 70, 1771-1777 (1991).

[6]. W.H. Press, S.A. Teukolsky, W.T. Vetterling and B.P. Flannery, "Numerical Recipes in C," 2nd ed., 397-402 (Cambridge Univ. Press, Cambridge, 1992). 